Rabaska

Revue d'ethnologie de l'Amérique française

\title{
La correspondance Barbeau-Tremblay
}

\section{Bertrand Bergeron}

Volume 13, 2015

Présence de Marius Barbeau : l'invention du terrain en Amérique française. Autour d'un legs centenaire (1914-2014)

URI : https://id.erudit.org/iderudit/1033759ar

DOI : https://doi.org/10.7202/1033759ar

Aller au sommaire du numéro

Éditeur(s)

Société québécoise d'ethnologie

ISSN

1703-7433 (imprimé)

1916-7350 (numérique)

Découvrir la revue

Citer cet article

Bergeron, B. (2015). La correspondance Barbeau-Tremblay. Rabaska, 13, 164-177. https://doi.org/10.7202/1033759ar

Ce document est protégé par la loi sur le droit d'auteur. L'utilisation des services d'Érudit (y compris la reproduction) est assujettie à sa politique d'utilisation que vous pouvez consulter en ligne.

https://apropos.erudit.org/fr/usagers/politique-dutilisation/ 


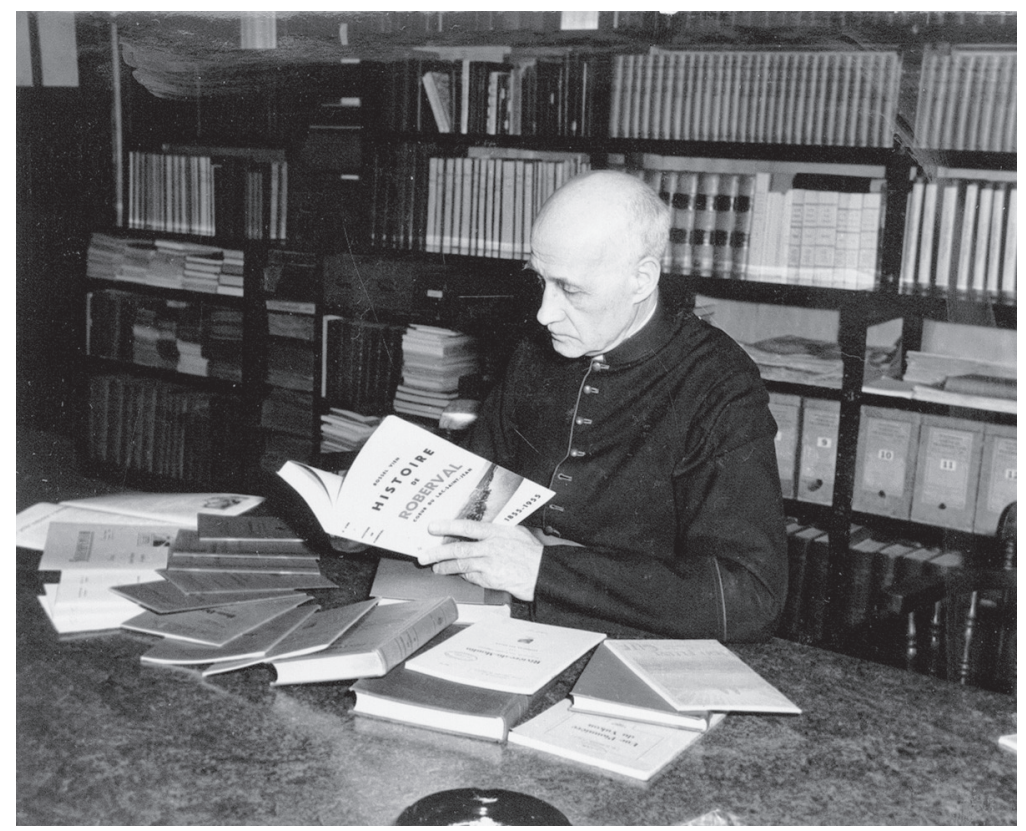

$M^{\text {gr }}$ Victor Tremblay (1892-1979)

Source : Fonds M ${ }^{\mathrm{gr}}$ Victor-Tremblay, Société historique du Saguenay. 


\title{
La correspondance Barbeau-Tremblay
}

\author{
BERTRAND BERGERON \\ Société québécoise d'ethnologie \\ J'ai des fils, disait l'autre, et ils grandissent \\ et je ne les aurai pas enseignés. \\ Je ne dépose donc rien en eux. \\ Et où irai-je une fois mort?
}

Antoine de Saint-Exupéry ${ }^{1}$

\section{Préambule}

Le projet de dépouiller la correspondance entre Marius Barbeau et Victor Tremblay m'est venu à la suite de l'annonce du colloque en l'honneur de l'éminent ethnologue. Lors d'un précédent colloque qui portait sur les prêtres collecteurs ${ }^{2}, \mathrm{j}^{\prime}$ avais découvert fortuitement, en fouillant dans le fonds $\mathrm{M}^{\mathrm{gr}}$ Victor-Tremblay à la Société historique du Saguenay, que Marius Barbeau lui avait prodigué des conseils pour l'instauration d'un musée régional ${ }^{3}$. Je n'avais pas poussé plus avant mes investigations, mon projet d'alors explorant un autre domaine de l'œuvre du père de l'histoire saguenéenne ${ }^{4}$.

La tenue de ce colloque m'offre l'occasion de revenir sur cette correspondance et de l'examiner en détail avec, pour toile de fond, la problématique des terrains d'enquête. Dans cet échange épistolaire, lequel du terrain de l'historien ou du terrain de l'ethnographe allait prédominer et imposer sa

1. Antoine de Saint-Exupéry, « Citadelle », Euvres, Paris, Gallimard, « La Pléiade », 1959, p. 546.

2. L'Apport des prêtres et des religieux au patrimoine des minorités. Parcours comparés Bretagne/Canada français. Actes des journées internationales d'étude tenues en 2011 à Pointe-del'Église (18-21 octobre) et à Brest (17-18 novembre), dans Port-Acadie. Revue interdisciplinaire en études acadiennes, $\mathrm{n}^{\text {os }}$ 24-25-26, automne 2013-printemps 2014-automne 2014, Université Sainte-Anne, $484 \mathrm{p}$.

3. «Si l'été prochain fait honorablement suite à l'hiver et au printemps merveilleux que nous avons au pays du Saguenay, écrit Victor Tremblay dans une lettre datée du 5 avril 1958, il faudra en profiter pour revoir notre Royaume. Je suis sûr que son musée saguenéen, dont l'existence est due à vos conseils, a ce qu'il faut pour vous retenir de longues heures, peut-être pour se faire qualifier de "trésor" régional. »

4. Bertrand Bergeron, «Les Prêtres et religieux historiens et promoteurs de sociétés d'histoire et de généalogie », L'Apport des prêtres et des religieux au patrimoine des minorités, op. cit., p. 273-289. 
démarche ? Même s'ils possèdent des accointances naturelles, la pratique de l'historien diffère sensiblement de celle de l'ethnographe, tout orientée qu'elle est vers la découverte et la consultation de documents fixés sur un support matériel accessible à merci : livre, mémoire, correspondance, etc.

En se privant volontairement de la documentation immatérielle, c'est-àdire celle qui repose tant sur la mémoire individuelle que collective, l'historien a pavé la voie à l'ethnographe dont le terrain est moins celui du dépôt d'archives que la fréquentation des représentants vivants d'une culture, d'un genre de vie qui s'observent et se décrivent dans la relation privilégiée avec des informateurs avertis et, d'une certaine manière, complices.

\section{Territoire et terrain}

Véritable rituel initiatique, le terrain constitue, pour l'ethnographe, le lieu physique où s'opère la confrontation entre un savoir acquis par l'enseignement et celui transmis par le geste et la parole. L'impétrant se trouve à la croisée des chemins : ou bien il cherchera à valider un savoir théorique, ou bien il apprendra à désapprendre sous la houlette de ses informateurs. Le fossé est parfois immense qui sépare la culture idéale de la culture vécue.

Si j'en parle en termes mystiques, c'est que le terrain est la condition indépassable du chercheur qui s'intéresse à la culture traditionnelle déclinée selon toutes ses composantes. Se confirmeront ou s'infirmeront des hypothèses, des présupposés ; d'autres naîtront du contact étroit et personnel avec des informateurs considérés comme les dépositaires d'un savoir qui se manifeste par des attitudes et des comportements ou s'expriment par des manières de dire et de narrer qui portent la patine de la tradition. Contrairement au colporteur qui écoule ses marchandises au hasard des habitations qui longent sa route, l'ethnographe refait le même chemin pour recueillir un savoir populaire afin de lui assurer la pérennité.

Dire de Marius Barbeau qu'il fut un homme de terrain est un pléonasme. De ce côté-ci de la Grande Mare, il en est l'inventeur. Au lieu de glaner selon son humeur et sa fantaisie avec l'esprit imprégné d'idées toutes romantiques, il introduisit dans la collecte des données rigueur et méthode, c'est-à-dire une démarche scientifique capable de valider les fruits de ses récoltes. Il y a, dans la pratique ethnographique, un esprit rustique qui l'apparente au moissonneur qui assure ses lendemains par son action d'aujourd'hui. Il amasse, engrange, pour mieux distribuer plus tard. Ses archives sont le grainier où la semence attend qu'une main vigilante la disperse avec « le geste auguste du semeur » magnifié par Victor Hugo.

Étrange occupation que de recueillir ce qui se brade à vil prix et se perd, chassé par un mode de vie en mutation accélérée. Que Marius Barbeau ait été l'inventeur du terrain d'enquête en Amérique française ne se discute 
plus, mais s'illustre en remontant dans le temps pour mettre nos pas dans les siens. Aux fins de l'exercice qui nous réunit dans ce colloque consacré à sa mémoire et à son œuvre, je m'en tiendrai à un aspect particulier de son action polyvalente : celle d'épistolier.

Son correspondant, Victor Tremblay, prêtre et historien, est né et a vécu au Saguenay-Lac-Saint-Jean. « Le Royaume du Saguenay » se hâterait-il de me corriger, territoire inventé par Donnacona au $\mathrm{XVI}^{\mathrm{e}}$ siècle pour satisfaire l'appétit aurifère et la curiosité de Jacques Cartier : «Car, rapporte celui-ci, il nous a certifié avoir été à la terre du Saguenay, où il y a infini or, rubis et autres richesses, et y sont les hommes blancs comme en France, et accoutrés de draps de laine ${ }^{5} »$. Pour la conscience européenne, ces propos rapportés situent d'emblée le Royaume du Saguenay comme un territoire imaginaire avant que d'être un territoire géographique. Ses frontières variaient au gré des descriptions qu'en donnaient les Amérindiens. «Il s'étendait depuis la rive du Saint-Laurent, entre l'embouchure de la rivière Moisie (en bas de Sept-Îles) et de l'île d'Orléans, jusqu'à une distance d'“une lune" de trajet vers l'ouest à partir de Tadoussac, ce qui reporte à une centaine de milles à l'ouest du lac Saint-Jean ${ }^{6} »$. Au Louvre, devant un auditoire avide de nouveautés mâtinées de merveilleux, le grand chef iroquois en rajoute : « Ce roi peau-rouge a raconté au roi de France que, au-delà de la grande rivière, se trouve une cité nommée Sagana, où il y a d'inépuisables mines d'or et d'argent, et où le peuple se vêt et porte des chaussures comme nous. En surplus, il y croît du poivre, du clou de girofle et de la muscade. [...] À la louange de cette riche nouveauté et rapportant d'autres contes, le roi ajouta que des hommes s'y rencontrent qui peuvent voler, ayant des ailes aux bras comme des chauvessouris $^{7} \gg$. Ces révélations fabuleuses n'échappèrent pas à l'oreille indiscrète de Legarto, espion de Charles Quint infiltré dans l'entourage de François $1^{\text {er }}$ qui en fit la relation à son roi. Donnacona eut la sagesse de dire au roi de France ce qu'il souhaitait s'entendre dire, ce qui est une manière éprouvée de se faire écouter et de recueillir l'adhésion de son entourage. On ne croit volontiers que ce qu'on est disposé à croire.

L'époque est à la découverte et aux extravagances cosmologiques. Les cordons des bourses se délièrent, on organisa d'autres expéditions. Le seul haut dignitaire de la cour de France à avoir jamais traversé l'océan y participa. Son aventure connut déboires et infortunes. Seule une ville du Lac-Saint-Jean garde la mémoire de son nom : Roberval.

5. Jacques Cartier, Voyages en Nouvelle-France, Montréal, Hurtubise HMH, « Cahiers du Québec », 1977, p. 124.

6. Victor Tremblay, « Le Nom de la région du Saguenay », Érudit : http://id.erudit.org/ iderudit/11358ac.

7. Marius Barbeau, Fameux Peaux-Rouges d'Amérique, Montréal, Beauchemin, 1966, p. 27. 
De 1534 à 1647, les natifs interdirent l'accès au Royaume du Saguenay. Il aura fallu la maladie d'une famille amérindienne convertie qui campait sur les rives du Piékouagami pour vaincre les réticences. L'honneur en revint au père Jean de Quen. La description de sa remontée du Saguenay en canot ramena l'aura fantastique qui auréolait le Royaume à des proportions plus humaines. À l'instigation des Charlevoisiens, 191 ans plus tard, le territoire imaginé s'enracina définitivement dans le réel en s'ouvrant à la colonisation. Arpenté, cadastré, peuplé, le territoire pouvait désormais servir de terrain pour d'éventuelles recherches. Par un étrange retour des choses dont la recherche est malgré tout coutumière, il arrive que le terrain, qui découle du territoire, devienne la condition de son réenchantement lorsque l'ethnographe recueille ce qui en est raconté par ses informateurs.

Dans toute entreprise de colonisation, il est un temps pour les pionniers et un autre pour ceux qui bénéficieront de leur labeur. Le défricheur précède le laboureur qui précède le citadin. Il faut une accumulation notable de capital pour que certains individus puissent se dégager des tâches serviles et orienter leur action vers les travaux de l'esprit. Ceux qui étudient une époque en sont rarement les contemporains. La mémoire familiale se déploie généralement sur trois générations (grands-parents, parents, enfants), parfois sur cinq si les enfants prennent consciencieusement le relais. Au-delà, le passé s'estompe dans un temps vague et brumeux. Comme historien, Victor Tremblay s'était rendu compte des trous qui béaient dans la documentation de sa région en raison de sa jeunesse même et des énergies mobilisées pour le défrichement. L'histoire était en partie orale, donc sujette à s'évanouir avec la succession des générations. Il y avait encore des témoins capables de remonter à la genèse de la région, mais leurs souvenirs étaient archivés dans l'immatérialité de leur mémoire. Pour pallier cette situation et sauver du naufrage des informations vitales pour l'identité collective, l'historien se fit homme de terrain et devint ethnographe en recueillant les souvenirs des vieillards. En consignant les réminiscences de ceux qui avaient connu la génération des pionniers, il servit d'interface entre la documentation orale et la documentation écrite, transposant la première en la seconde qui devint une source inépuisable d'informations pour des historiens qui ne jurent que par les sources écrites. À cet effet, il rédigea un questionnaire qu'il remit à ses élèves afin qu'ils puissent relever avec profit les témoignages de leurs aînés. Grâce à cette vaste anamnèse collective, Victor Tremblay sauva de l'oubli un patrimoine immatériel inestimable.

C'est à cette époque que Marius Barbeau visita le Saguenay. À son habitude, il explora certains sites, consulta les archives disponibles dont celles de la jeune Société historique du Saguenay. L'homme qui venait à peine de 
publier The Kingdom of Saguenay ${ }^{8}$ était fait pour s'entendre avec celui qui était en train d'écrire L'Histoire du Saguenay ${ }^{9}$. Tous deux se souciaient de la place dévolue aux humbles dans la genèse et l'évolution de l'aventure humaine. Ils étaient convaincus que l'heureuse conjonction du terrain de la culture et de celui de l'histoire produit la civilisation, territoire spirituel qui ouvre à l'homme le champ de ses possibles. Il n'est qu'à méditer les paroles de Saint-Exupéry pour s'en persuader : « J'ai vu des danseuses composer leur danse. Et la danse une fois créée et dansée, certes personne n'emportait le fruit du travail pour en faire des provisions. La danse passe comme un incendie. Et cependant je dis civilisé le peuple qui compose ses danses, malgré qu'il ne soit pour les danses ni récoltes ni greniers. Alors que je dis brute le peuple qui aligne sur ses étagères des objets fussent-ils les plus fins, nés du travail d'autrui, même s'il se montre capable de s'enivrer de leur perfection ${ }^{10}$. "

L'historien avait besoin des conseils de l'ethnologue. II en résulta une correspondance qui s'échelonna sur vingt-cinq ans, soit de 1937 à 1962.

\section{Considérations matérielles}

Conscient de l'importance de la société d'histoire qu'il venait de fonder et de son rôle de pionnier, Victor Tremblay a pris un soin méticuleux à archiver cette correspondance, conservant une copie de ses propres lettres. Au total, nous nous retrouvons devant un lot de 151 lettres et quelques notes réparties comme suit : 83 lettres de Victor Tremblay et 68 lettres de Marius Barbeau.

Dans la quasi-totalité des cas, elles ont été rédigées sur du papier format lettre (8,5 x 11). Celles de Victor Tremblay sont toutes dactylographiées et paraphées avec la mention prêtre abrégée (ptre) ; celles de Marius Barbeau sont manuscrites dans $40 \%$ des cas, soit 28 sur 68 et signées. Victor Tremblay écrit depuis le séminaire de Chicoutimi qui héberge la Société historique. Dans la plupart des cas, Marius Barbeau utilise le papier à en-tête du National Museun of Canada, institution qui dépend, au début de la correspondance, du Department of Mines and Resources, Mines and Geology Branch. Plus tard, au cours de la correspondance, le papier à en-tête nous apprendra le changement d'affiliation et la francisation de l'organisme : Musée National du Canada, Division des Parcs Nationaux, Ministère des Ressources et du Développement Économique (lettre datée du 18 avril 1951). C'est ainsi que l'évolution d'une société s'enregistre indirectement dans son courrier officiel.

8. Marius Barbeau, The Kingdom of Saguenay, Toronto, The Macmillan Co. of Canada, 1936, 167 p. Ce livre a été traduit en français sous le titre de Le Saguenay Légendaire, Montréal, Librairie Beauchemin Limitée, 1967, 147 p.

9. Victor Tremblay, L'Histoire du Saguenay depuis les origines jusqu'en 1870, Chicoutimi, La Librairie Régionale Inc., 1968, 465 p. Cette édition reprend, pour l'essentiel, celle de 1938.

10. Antoine de Saint-Exupéry, " Citadelle », Euvres, Paris, Gallimard, « La Pléiade », 1959, p. 542 . 
Sérinalre de Chlcoutimi, le s ootobre 1937.

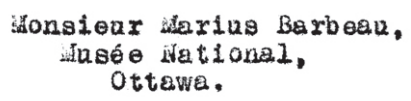

Bien chor hansion.

Je suls heurbux a vous adreser ane copte de notro version de la "Charion des voyageurs".

Je vola aaresse on même temps une cople de la carte bistoriqae que noue avons preperés ot qui a ete imprimé 10 jour même de votre depert de Chlcontinh. Vous la rocevez है t1tre de wenbre de le socíte Historiquo du Saguanay.

Hous cropons avolr mis la mein sur l'home qu'il nous faut pour pousser lo travell intsrassant ot utile dont vous hous avaz donné l'idée netto. iọus annonoe dos résultets substent lels. it j'ospore quo nous anxons quelque chose \& vous montrar lors de votre nouvolle visite su "Royaume die Saguenay".

J'al trouve ot acquis une pibe an terre cuite -.. petit suvonaler-m- exécuté per Mapoléon Trambiay et jo suis sur le vole pour en découvrix dautros. 81 nous reuselssono une coejilette en co domalno, hous vous perons votro part. Il soable que Delislo ait été le nojlietra de nos potiers. Le travail de irenolay (colui que nousevons) est bien fait. mais il a'est pas reluarquablo. On ne peut pas toutefols fifger par cotte soule pioco, qui d'eilleurs no conmadalt pas uno application spéciale.

J'aurai blentôt des indications ar los Porgerons qul ont exécuté los orolx do cluetiores que vous avoz renarQue Q

Espérant quo los souvenira que rous gardoz de votro "découverto du saguensy" vaus y remeneront, et on attendent vous y attacheront. Je vous prie d'agréer l'expression do mes meilieurs sentimente.

$$
\begin{array}{r}
\text { Bion coralalement a vous. } \\
\text { V. The tre }
\end{array}
$$

\section{Lettre tapuscrite de Victor Tremblay à Marius Barbeau} depuis Chicoutimi datée du 5 octobre 1937.

Source : Fonds $\mathrm{M}^{\mathrm{gr}}$ Victor-Tremblay, Société historique du Saguenay. 
NATIONAL MUSEUM OF CANADA

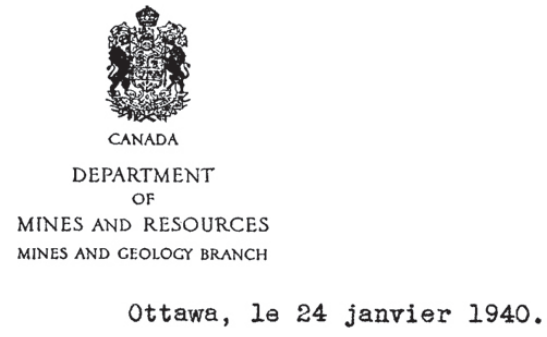

Ottawa, le 24 janvier 1940.

\begin{abstract}
Monsieur l'abbé Victor Tremblay, Séminaire de Chicoutimi, Chicoutimi, zuébec.

Cher monsieur l'abbé:

Je vous remercie bien sincèrement des renseignements précis et intéressants que vous me donnez sur l'industrie du sucre d'érable dans le Saguenay. Je m'en servirai bientôt au cours de ma prochaine étude sur ce sujet.

Je viens de lire dens un journal dernièrement qu'on vous avalt consulté au sujet de certain's ossements trouvés à la Rivière-du-Moulin. Pouvez-vous me dire ce qu'on entend par un cercueil de bois tressé (I'Evènement, 19 janvier 1940)? On dit que certains ossements étaient d'une sauvagesse. Dans ce cas, s'ils sont intéressants à aucun autre, je suis sûr qu'ils seraient bienvenus dans les collections du Musée national, si on peut obtenir ces restes sans trop d'embarras.

Bien sincèrement,

$$
\text { Manis Batean }
$$

$\mathrm{MB} / \mathrm{JB}$

Marius Barbeau.
\end{abstract}

\title{
Lettre tapuscrite de Marius Barbeau à Victor Tremblay depuis Ottawa datée du 24 janvier 1940.
}

Source : Fonds M ${ }^{\mathrm{gr}}$ Victor-Tremblay, Société historique du Saguenay. 
DeuXIÈME PARTIE

Bertrand Bergeron

$1 x$

NATIONAL. MUSEUM OF CANADA

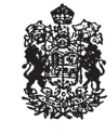

CANADA

DEPPARTMENT

OF

MINES AND RESOURCES

MINES AND GEOLOCY BRANCH

OHaiva, 27 agyo

Cher Mesesiren, Trembla,

Oc sus remena's Jo volu denicielethe. Snivaut

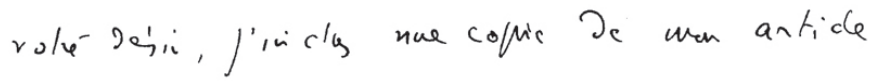
ar il at pustion Je l'ant Des jecunes "prinityfs" de la ralbaic.

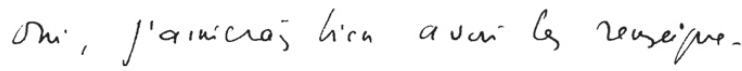

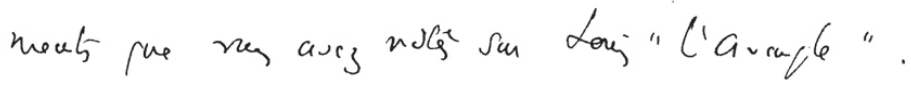

Sn vees salvant bisù sivicéverus

reans Gabrem

Lettre manuscrite de Marius Barbeau à Victor Tremblay depuis Ottawa, datée du 27 février 1940.

Source :Fonds M ${ }^{\mathrm{gr}}$ Victor-Tremblay, Société historique du Saguenay.

172

RABASKA 
Pour anodins que paraissent ces détails, ils n'en sont pas moins révélateurs du caractère des correspondants. Victor Tremblay, homme de hiérarchie et de devoir, ne déroge pas de la forme conventionnelle apprise.

À quelques nuances près, ses entrées et ses sorties sont identiques et dénotent, à travers cette étiquette à la politesse empesée, à la fois la déférence envers un interlocuteur qui occupe un poste important dans une institution prestigieuse et le caractère ordonné, discipliné, voire sévère du rédacteur en ce qu'il démontre un respect inné envers la hiérarchie. Le père de l'histoire du Saguenay-Lac-Saint-Jean n'est pas sans savoir que le titulaire d'une haute fonction peut exercer une influence décisive quand le besoin s'en fait sentir. Victor Tremblay évolue au sein de l'Église catholique et progresse à l'intérieur de sa nomenclature, passant du statut de prêtre séculier à celui de chanoine et de prélat domestique. Ces nominations lui sont une source de satisfaction personnelle et il s'en montre fier.

Cette manière presque scolaire de s'adresser à Barbeau signale en même temps la relation qui s'établit naturellement entre le disciple et le maître : la correspondance se déroule sur le terrain de l'ethnographie, discipline dans laquelle Barbeau excelle. Ces marques de respect et de déférence reviennent à plusieurs reprises : «J'apprécie grandement votre attention, écrit Tremblay le 19 mars 1944, à tout ce qui peut aider nos humbles efforts ; c'est un puissant encouragement en même temps qu'un concours toujours utile ». Il récidive le 19 mars 1946 : «J'apprécie grandement vos conseils ».

Au début de la correspondance, Barbeau emploie lui aussi des formules protocolaires : «Cher monsieur Tremblay ». Au fur et à mesure des envois, le ton se réchauffe. Il met en valeur les titres honorifiques de son correspondant : abbé, chanoine, monseigneur, révérend père. Vers la fin, il y va franchement d'un « très cher ami », ce que Victor Tremblay n'ose se permettre. Quand il semble pressé par le temps, il répond avec cette écriture griffonnée qui court sur la feuille. Apprenant la nouvelle de la récente nomination du chanoine Tremblay au titre de prélat domestique, Barbeau le félicite et termine par une exclamation enthousiaste : "Vive le Royaume de Saguenay! (5 décembre 1957). Ce cri du cœur a dû émouvoir le destinataire, lui qui a voué sa vie à promouvoir et à défendre bec et ongles l'appellation « Royaume du Saguenay».

\section{De quoi parlent-ils ?}

Dans les relations humaines, le silence sur certains sujets en révèle autant que les considérations sur d'autres. Une correspondance qui s'étale sur vingt-cinq ans s'inscrit dans la durée et, partant, dans l'histoire. Les trois grands absents de cette relation épistolaire sont d'ordre social, personnel et intellectuel.

De 1937 à 1962, des événements tragiques ont secoué brutalement la planète : Deuxième Guerre mondiale, Shoah, guerre de Corée, guerre du 
Vietnam, effervescence nationale dans la foulée de la Révolution tranquille, etc. Or, rien de ces événements ne vient altérer la sérénité olympienne des échanges épistolaires. Un mot permet de présumer que l'écho du conflit mondial résonne aux oreilles du paisible prélat : «J'espère que l'été 1942, confie-t-il le 7 juin 1942, va vous permettre de vous éloigner de la Capitale et du bombardement de besognes qui vous assaillent ». La seule guerre qui présente quelque intérêt factuel est celle de Crimée qui s'est livrée un siècle plus tôt au motif qu'elle a enrichi la toponymie du Saguenay-Lac-Saint-Jean : «Vous savez sans doute l'étude faite par M. Wintemberg sur les noms géographiques échos de la guerre de Crimée au Canada. J'ai pu y ajouter deux noms de la région du Saguenay, ignorés de l'auteur : $A \operatorname{lma} a^{11}$ (donné à une île, puis à une localité que vous connaissez) et Redan (appliqué à une région ravinée près de Chicoutimi) » (lettre du 29 avril 1943). C'est tout. Barbeau ne saisit pas le prétexte de cette information pour glisser un commentaire sur le conflit actuel qui sévit dans la même région.

Les deux chercheurs œuvrent dans un univers feutré, dans un sanctuaire que les enjeux des théâtres d'opérations ne viennent pas troubler. Ils évoluent dans un temps anhistorique, un hors-temps qui leur épargne les vicissitudes de leur époque. Deux intellectuels totalement absorbés par leur quête devisent paisiblement au pied d'un volcan en éruption. D'aucuns pourraient penser que c'est là faire preuve d'insensibilité caractérisée. Pourtant, il n'en est rien. Ils agissent comme agissent les personnages de Pieter Brueghel l'Ancien dans son célèbre tableau La Chute d'Icare. Chacun vaque à ses occupations coutumières pendant qu'Icare s'enfonce dans la mer dans l'indifférence générale. Les témoins potentiels n'ont pas fait preuve de dureté de cœur ; ils étaient requis par des travaux qu'ils jugeaient urgents, et le drame leur a tout bonnement échappé. Les hommes font l'histoire, écrivait en substance Raymond Aron, mais ils ne savent pas l'histoire qu'ils font.

Les deux intellectuels n'évoquent pour ainsi dire jamais les événements touchant leur vie personnelle. C'est de manière fortuite que l'on apprend le séjour de Victor Tremblay dans un hôpital montréalais ou l'interdiction faite à Marius Barbeau par son médecin de fréquenter son bureau pour deux mois. L'un et l'autre ne dévoilent ces détails que pour excuser un retard dans leur correspondance. Le seul élan vraiment spontané est redevable à Barbeau quand il félicite son correspondant pour sa promotion. C'est avec un «Vive le Royaume de Saguenay ! » enthousiaste qu'il termine sa lettre. On imagine bien que les deux interlocuteurs savaient des choses l'un sur l'autre par des voies latérales que leur discrétion leur interdisait de mentionner. Devait entrer dans cette disposition d'esprit une bonne part de respect humain réciproque.

11. L'auteur souligne les deux noms. 
La même attitude colore leur activité professionnelle. On ne décèle chez eux aucune divergence intellectuelle. En vertu d'un accord tacite, évitaientils soigneusement toute problématique qui les aurait conduits inévitablement à s'affronter? Je ne le pense pas. Sur une si longue durée, des divergences d'opinions, si elles avaient existé, auraient fini par percer dans leurs écrits. Cette attitude sereine trouve son explication dans la nature même de leur relation : ce sont deux hommes de terrain au caractère pragmatique qui s'emploient à résoudre des problèmes pratiques dans lesquels aucune école de pensée ne vient interférer. Devant chacun d'eux se déploie un chantier gigantesque qu'il est important de gérer. Tout est à faire et seuls comptent pour le moment les problèmes d'intendance. Aux générations suivantes est laissé le soin de s'interroger sur des problèmes classificatoires. Ils organisent leurs actions. Viendra une génération qui s'attachera à la penser.

Certains commentateurs de Barbeau ont voulu voir dans sa démarche des relents de l'école évolutionniste qui avait cours, chez certains anthropologues, dans le premier quart du $\mathrm{xx}^{\mathrm{e}}$ siècle. Aucun indice décelable ne permet d'appuyer cette thèse, du moins dans la correspondance que nous avons sous les yeux.

Les deux chercheurs ne s'écrivent que pour des raisons professionnelles. Si on se réfère au fonds conservé à la Société historique du Saguenay, l'initiative de celle-ci reviendrait à Victor Tremblay en réponse au désir de son illustre visiteur de recevoir la «Chanson des voyageurs » (lettre datée du 5 octobre 1937). Tout donne à penser qu'à cette occasion, Victor Tremblay a servi de guide à son hôte lors d'une visite des cimetières de Sainte-Annede-Chicoutimi et de Saint-Fulgence. Barbeau travaillait, à ce moment-là, sur les artisans du fer et procédait à un relevé des croix en fer forgé. Dès la deuxième lettre, Tremblay fournit les noms des auteurs des croix qui ont été signalées à son attention.

D’emblée, le ton est donné. Barbeau reste fermement campé sur le terrain de l'ethnographie; Tremblay quitte celui de l'histoire pour le rejoindre sur le sien. On va discuter de culture traditionnelle et non d'histoire. Cette dernière ne sera requise que pour des considérations ancillaires. Ce qui balisera ce long parcours qui s'étendra sur vingt-cinq ans, ce sont les préoccupations professionnelles de Barbeau, épisodiquement, celles de Tremblay. Il arrivera assez souvent au premier de servir de personne ressource pour identifier et classifier tel ou tel artefact (un retable découvert au monastère de Mistassini, par exemple). En retour, Tremblay se livrera à des compléments d'enquête pour son correspondant, sera consulté pour préciser tel ou tel détail d'une étude en cours (sur la poterie, par exemple) pourvu que la région soit concernée par l'objet de la recherche. Auquel cas, il se dépensera sans compter, anticipant parfois certains points à être précisés et, marque d'estime signalée, Barbeau 
le priera de lire le manuscrit d'une étude en cours de rédaction afin de lui suggérer des corrections, s'il y a lieu. Flatté par cette reconnaissance de ses compétences professionnelles, Victor Tremblay s'acquittera avec empressement de ce service, joignant à sa réponse une série de notes pour lesquelles il est chaleureusement félicité.

D'aucuns pourraient croire que la notoriété de Barbeau lui a permis d'inféoder Tremblay. Opiner en ce sens fausserait la perspective de leurs relations. Il est certain que Barbeau manœuvrait en terrain conquis et que Tremblay pouvait laisser penser qu'il assurait l'intendance. Mais ce dernier savait tirer son épingle du jeu avec doigté. Il n'hésitait pas à requérir l'aide de l'éminent ethnologue dans des dossiers névralgiques pour sa jeune société : les fouilles archéologiques laissées pendantes à Tadoussac et celles à entreprendre de toute urgence au poste de traite à l'embouchure de la Métabetchouan où ne subsistait plus qu'une poudrière. Il faut comprendre qu'en regard du National Museum of Canada, immensément mieux pourvu en ressources humaines et matérielles, la jeune Société historique du Saguenay faisait figure de parent pauvre. Les compétences professionnelles lui manquaient dramatiquement. Dans une lettre datée du 24 mai 1938, il s'en ouvre avec franchise : « Je vous rappelle tout l'intérêt historique qu'il y aurait à faire des fouilles sur le site de l'ancien Poste de traite à l'embouchure de la rivière Métabetchouan, où plus de 300 ans d'activité intense ont dû enfouir une foule de choses retrouvables. [...] Il suffirait donc d'avoir un expert comme celui que vous nous avez recommandé. [...] J'écris à l'instant même à la Commission des Monuments Historiques. Je compte sur vous pour dire le bon mot et donner la bonne poussée afin qu'on sache que la chose en vaut la peine et qu'on la prenne au sérieux $»$.

Il est paradoxal de constater la part congrue laissée à la culture immatérielle dans cette correspondance. Outre cette " Chanson des voyageurs" inaugurale, on n'y trouve mention que d'Alexis le Trotteur et de Louis l'Aveugle, deux personnages emblématiques du Saguenay légendaire et tous deux originaires de Charlevoix. Le Saguenay de Barbeau se révèle plus près de son étymologie amérindienne (« eau qui sort» pour se mêler à celle de « la route qui marche », autre nom du Saint-Laurent) que celui de Tremblay qui s'étend jusqu'en Abitibi.

Ce peu de cas fait à la culture immatérielle peut s'expliquer à la fois par les préoccupations ponctuelles de Barbeau et la formation de son correspondant saguenéen : un historien n'avance jamais que sur une route pavée de documents déposés sur un support matériel. Nous avons souligné, plus haut, l'ambition de Victor Tremblay de transformer une mémoire orale (les dires des vieilles gens) en documents écrits au moyen de cueillettes intensives et uniformisées. Barbeau ne manque pas de signaler la parution de ses écrits, 
notamment, dans sa lettre du 16 octobre 1946, de Tall Tales of the Saguenay qui brosse le portrait de quelques « originaux et détraqués », pour reprendre le titre d'une œuvre de Fréchette : «Alexis the Horse - Boily the bone-setter - The Yellow-haired Potter (Belleau) - The Blind Singer (Louis l'Aveugle) - The Village Crier (J.B. Côté)».

L'éventail des sujets abordés est impressionnant : orfèvrerie, poterie, statuaire, sculpture, peinture, sucre d'érable, archéologie, ébénisterie, tissage, teinture végétale, etc. Rien des aspects de la culture matérielle n'est étranger à l'attention méticuleuse des deux chercheurs. Il est dans la nature même des pionniers de se dépenser tous azimuts parce que tout est à faire à la fois et en même temps dans l'urgence de ne rien oublier de ce qui mérite d'être sauvé.

\section{Conclusion}

Au terme de leur correspondance, Barbeau et Tremblay auront blanchi sous le harnais pendant un quart de siècle. La retraite venant, ils eurent de moins en moins de raisons professionnelles de s'écrire. Aussi leurs lettres s'espacèrent-elles. Elles prirent fin abruptement, c'est du moins ce qu'autorise à croire les traces qui en sont conservées dans le fonds $\mathrm{M}^{\mathrm{gr}}$ Victor-Tremblay.

À cette étape de notre investigation, il est loisible de se demander quel portrait ces quelque 150 lettres ont brossé de leurs auteurs.

D'abord l'image de deux êtres pleinement conscients de la place qu'ils vont occuper dans l'histoire, de deux passionnés par leur discipline, de deux intellectuels polyvalents attentifs au moindre détail, précis dans leurs observations, de deux chercheurs pragmatiques qui fuient toute polémique en ne s'embarrassant pas de théorie, mais en collant aux faits, de deux hommes intègres qui n'hésitent pas à rendre à chacun son dû, qui s'admirent avec l'humilité que confère la grandeur authentique.

Barbeau a laissé des archives débordantes qui renferment l'âme autant des Canadiens français que des peuples autochtones. Tremblay a légué une société d'histoire richement documentée qui a permis l'élaboration d'une maîtrise en histoire régionale dispensée par l'U $\mathrm{QAC}^{12}$.

Quand je considère ces deux destins exceptionnels, je ne puis m'empêcher de penser à cet éloge des commencements qui termine Tristes Tropiques de Claude Lévi-Strauss : «L'homme ne crée vraiment grand qu'au début; dans quelque domaine que ce soit, seule la première démarche est intégralement valide. Celles qui suivent barguignent et se repentent, s'emploient, parcelle après parcelle, à récupérer le territoire dépassé ${ }^{13} .{ }^{14}$

12. Université du Québec à Chicoutimi.

13. Claude Lévi-Strauss, Tristes Tropiques, Paris, Plon, 1955, p. 489.

14. Je remercie $\mathbf{M}^{\mathrm{me}}$ Marie-Josée Gagné de la Société historique du Saguenay d'avoir reprographié la correspondance Barbeau-Tremblay à mon intention. 